\title{
Discours
}

Revue de linguistique, psycholinguistique et

informatique. A journal of linguistics, psycholinguistics and computational linguistics

26 | 2020

Varia

\section{L'expression du déplacement en italien et français L2 : influence translinguistique $v s$ tendances communes}

\section{Simona Anastasio et Sandra Benazzo}

\section{OpenEdition}

\section{Journals}

Édition électronique

URL : https://journals.openedition.org/discours/10736

DOI : $10.4000 /$ discours.10736

ISSN : 1963-1723

\section{Éditeur :}

Laboratoire LATTICE, Presses universitaires de Caen

\section{Référence électronique}

Simona Anastasio et Sandra Benazzo, «L'expression du déplacement en italien et français L2

influence translinguistique vs tendances communes », Discours [En ligne], 26 | 2020, mis en ligne le 02 novembre 2020, consulté le 06 février 2023. URL : http://journals.openedition.org/discours/10736 ; DOI : https://doi.org/10.4000/discours. 10736

\section{(c) (i) $\odot$}

Creative Commons - Attribution - Pas d'Utilisation Commerciale - Pas de Modification 4.0 International - CC BY-NC-ND 4.0

https://creativecommons.org/licenses/by-nc-nd/4.0/ 

Revue de linguistique, psycholinguistique et informatique

\section{L'expression du déplacement en italien et français L2: influence translinguistique vs tendances communes}

Simona Anastasio

UMR 7023 Structures formelles du langage

CNRS, Université Vincennes-Saint-Denis - Paris 8

Sandra Benazzo

UMR 7023 Structures formelles du langage

CNRS, Université Vincennes-Saint-Denis - Paris 8

Simona Anastasio, Sandra Benazzo, «L'expression du déplacement en italien et français L2: influence translinguistique vs tendances communes », Discours [En ligne], 26 | 2020, mis en ligne le 2 novembre 2020.

URL: http://journals.openedition.org/discours/10736

Titre du numéro: Varia

Coordination: Nicolas Hernandez \& Lydia-Mai Ho-Dac

Date de réception de l'article: 16/12/2019

Date d'acceptation de l'article: 10/04/2020 



\title{
L'expression du déplacement en italien et français L2 : influence translinguistique vs tendances communes
}

\author{
Simona Anastasio \\ UMR 7023 Structures formelles du langage \\ CNRS, Université Vincennes-Saint-Denis - Paris 8 \\ Sandra Benazzo \\ UMR 7023 Structures formelles du langage \\ CNRS, Université Vincennes-Saint-Denis - Paris 8
}

\begin{abstract}
Nous présentons dans cet article une étude transversale sur l'expression du déplacement dans le discours narratif oral chez des apprenants de l'italien et du français langues secondes (L2), ayant comme langue maternelle (L1) soit une langue typologiquement proche (italien ou français) soit une langue distante (anglais) de la variété cible. Ces combinaisons de langue source-langue cible (LS-LC) visent à vérifier l'étendue de l'influence translinguistique et son évolution en fonction du niveau de compétence de la L2 (intermédiaire vs avancé). Si des variations inter-typologiques sont attestées entre les locuteurs natifs de l'anglais vs ceux du français et de l'italien, des différences intra-typologiques sont également identifiables dans la distribution de l'information de la trajectoire (locus) au sein d'une proposition entre les deux langues à cadrage verbal considérées. Les données non natives relèvent des tendances communes attribuables aux principes généraux d'apprentissage dans les récits des apprenants intermédiaires (c'est-à-dire emploi de formes verbales et prépositionnelles idiosyncrasiques, mais pas de différences liées à la L1). Certaines divergences liées à l'influence de la L1 sont aussi attestées mais surtout aux stades avancés, lorsque l'apprenant maîtrise un vocabulaire assez diversifié pour transférer des structures typiques de la L1 en L2 et si l'input favorise ce type de transfert.
\end{abstract}

Mots clés: discours oral, situations de déplacement, parcours développemental, influence translinguistique, acquisition $\mathrm{L} 2$

This paper presents a cross-sectional study of the expression of motion events in oral narrative discourse produced by second language (L2) learners of Italian and French, whose first language (L1) is either typologically close (Italian or French) or distant (English) from the target language. This combination of source and target languages aims at verifying the impact of crosslinguistic influence and its development with respect to $L 2$ proficiency level (intermediate vs. advanced). While strong intertypological variations are attested between English vs. French and Italian speakers in their native language, some intratypological variation is also found between the two verb-framed languages in question concerning the distribution of path information within a sentence (locus). The analysis of non-native data reveals some common tendencies related to general acquisitional principles in the productions of intermediate learners (e.g., use of idiosyncratic verb and prepositional forms, but no specific L1 effect in L2). Some differences related to L1 influence are attested but only at the advanced level, i.e. when the learner has developed a vocabulary rich enough to transfer L1 structures to L2 discourse, and only if the input favours this phenomenon.

Keywords: oral discourse, motion events, developmental trajectory, crosslinguistic influence, second language acquisition (SLA) 


\section{Introduction}

L'espace est un domaine fondamental de la cognition humaine qui a fait l'objet de nombreuses études notamment à cause de la spécificité de ses composantes et de leur distribution variable à travers les langues. En effet, malgré la possible universalité des concepts relatifs à l'espace (voir Levinson, 2003), les langues diffèrent fortement dans les moyens linguistiques qu'elles offrent à leurs locuteurs pour l'expression du type des relations spatiales relatives aux événements spatiaux (de localisation, de déplacement, etc.). L'étude de son expression en langue seconde (L2) présente un intérêt particulier, notamment concernant la capacité de l'apprenant à restructurer les concepts spatiaux typiquement encodés dans sa langue maternelle (L1) dans le processus de rapprochement à la langue cible (LC) que l'apprentissage d'une L2 implique.

À l'intérieur des relations spatiales, notre étude se focalise sur l'expression des situations de déplacement (motion events), à savoir des situations qui impliquent le changement de localisation d'une entité d'un point $X$ à un point $Y$ dans un certain intervalle temporel (Talmy, 1985). Lorsqu'un locuteur décrit ce type de situation il peut décider de se référer à la trajectoire ${ }^{1}$ (path, voir Talmy, 2000) suivie par l'entité en mouvement (figure) dans sa phase initiale (source) et/ou finale (but pour Borillo, 1998) du déplacement, à la manière dont cette entité se déplace et éventuellement à la cause provoquant ce déplacement. La trajectoire est cependant considérée comme la composante fondamentale et universelle des situations de déplacement, pouvant s'exprimer de différentes manières à travers les langues (par ex. dans le verbe principal et/ou dans la périphérie verbale d'un énoncé). La classification typologique des langues proposée par Talmy (1985 et 2000) est en effet basée sur le locus d'encodage de la trajectoire. Dans les langues à cadrage satellitaire (par ex. les langues germaniques et slaves), la trajectoire est typiquement encodée dans des particules directionnelles associées au verbe, dites satellites, tandis que la manière et/ou la cause du déplacement sont lexicalisées dans la racine verbale (ang. run [Manière] out [Trajectoire]). Inversement, dans les langues à cadrage verbal (par ex. les langues romanes) la trajectoire est exprimée dans le verbe, alors que d'autres composantes, telles que la manière ou la cause, sont soit omises soit encodées dans la périphérie du verbe principal, par exemple dans des propositions subordonnées, des gérondifs, etc. (fr. partir [Trajectoire] en courant [Manière]).

Néanmoins, de nombreuses études montrent que certaines langues ne peuvent s'inscrire ni dans les langues à cadrage verbal ni dans les langues à cadrage satellitaire parce qu'elles présentent plutôt des stratégies de lexicalisation mixtes ou se situant à mi-chemin entre les deux groupes (voir Cini, 2008, pour l'italien). Par ailleurs, à côté des différences inter-typologiques mentionnées (cadrage verbal vs satellitaire), il existe également une certaine variation intra-typologique entre langues appartenant

1. Nous adoptons la définition de trajectoire fournie par Talmy (2000), à savoir le parcours suivi par l'entité en mouvement. Ce parcours se constitue de trois sous-composantes: les différents types de trajectoire («vecteur», source, but), la conformation géométrique de la trajectoire et la deixis. Nous nous focaliserons uniquement sur la première sous-composante. 
à la même famille du point de vue de l'expression du déplacement (voir IbarretxeAntuñano, 2009 et 2015, Ibarretxe-Antuñano et Hijazo-Gascón, 2012, pour le basque vs l'espagnol, deux langues à cadrage verbal; Ragnasdóttir et Strömqvist, 2004, pour l'islandais vs le suédois, deux langues à cadrage satellitaire).

En ce qui concerne les langues romanes, pour certaines situations de déplacement, notamment celles impliquant un franchissement de frontière, l'italien permet de choisir entre les deux types de lexicalisation du mouvement. Par exemple, l'action de sortir peut être encodée par uscire (construction à cadrage verbal) ou venir fuori (construction à cadrage satellitaire). Les constructions analytiques comme venir fuori, typiques de l'oral, correspondent à la catégorie des verbes syntagmatiques ${ }^{2}$ (VS - Simone, 1997 et 2008; Iacobini et Fagard, 2011) associant des verbes de déplacement (comme andare [aller]) et/ou de manière (comme correre [courir]) à des particules directionnelles - telles que fuori (trajectoire de l'intérieur vers l'extérieur), dentro (trajectoire de l'extérieur vers l'intérieur), via (trajectoire d'éloignement) - qui ressemblent fortement aux constructions satellitaires à particules de l'anglais. Grâce à leur emploi, la trajectoire peut se décrire de manière plus détaillée en italien qu'en français (Anastasio, 2017 et 2018) ou en espagnol (Ibarretxe-Antuñano et Hijazo-Gascón, 2012 et 2013). Le français aussi, de son côté, semble en quelque sorte présenter un système typologiquement hybride (Kopecka, 2006) ou opaque (Hendriks et Hickmann, 2011) en raison de sa distribution variable des informations spatiales au sein d'une proposition. En effet, bien que le français soit une langue à cadrage verbal, dans les situations de mouvement provoqué la trajectoire peut être exprimée par des éléments extraverbaux, laissant ainsi la racine verbale libre pour l'encodage de la cause et ou de la manière (par ex. «il a tiré [Cause + Manière] le sac très lourd tout en baut du toit [Trajectoire]», Hendriks et al., 2008: 28). À la lumière de tels résultats issus de recherches plus récentes, bon nombre de chercheurs préfèrent situer les langues sur un continuum de saillance des composantes spatiales au lieu de les classer dans des catégories binaires (voir Slobin, 2006; Cadierno et Ruiz, 2006; Kopecka, 2006 et sous presse; Ibarretxe-Antuñano, 2009; Spreafico, 2008 et 2009; Beavers et al., 2010; Hendriks et Hickmann, 2011; Verkerk, 2015; Soroli et Verkerk, 2017; Anastasio, 2018).

Par ailleurs, il a été montré que les spécificités typologiques ainsi décrites ont un impact sur la manière dont les locuteurs conceptualisent les composantes spatiales à exprimer pour décrire une même situation. Les travaux de Slobin (hypothèse du Thinking for Speaking [Penser pour Parler], Slobin, 1996) mettent en évidence les différents choix des locuteurs des langues à cadrage verbal $v$ s satellitaire, pour encoder le même type de déplacement, en fonction des moyens qui sont facilement

2. Simone (1997) définit les verbes syntagmatiques (VS) comme «des syntagmes composés d'une tête verbale et d'un complément constitué d'une particule (à l'origine un adverbe) unis par une cohésion syntaxique à un degré tel qu'il n'est pas possible de commuter le VS entier avec un seul de ses éléments» (Simone, 1997: 49, traduit par Moncó Taracena, 2013: 65). Cette classe lexicale est très répandue dans l'usage de la langue italienne et distingue celle-ci des autres langues romanes du point de vue de la typologie lexicale (Schwarze, 1985). 
accessibles et fréquemment utilisés dans leur L1: la disponibilité de ces moyens attire l'attention du locuteur vers l'expression de certains aspects du déplacement au détriment d'autres (Berman et Slobin, 1994). À titre d'exemple, les locuteurs des langues satellitaires tendent à exprimer la manière du déplacement plus souvent et de façon plus détaillée que les locuteurs des langues à cadrage verbal, parce que leur langue met à disposition un lexique très diversifié de verbes de manière. Parallèlement, le lexique correspondant des langues à cadrage verbal est moins diversifié. Pour encoder la manière, leurs locuteurs doivent recourir à des constructions plus complexes - dont des propositions subordonnées - impliquant un coût cognitif plus important, ce qui les amène à omettre cette information si elle n'est pas fondamentale pour la cohérence du discours (Berman et Slobin, 1994; Slobin, 2004 et 2006).

Les travaux sur l'acquisition de la L1 ont montré l'enracinement progressif, chez l'enfant, de la perspective discursive typiquement adoptée par les adultes de la même langue (voir Berman et Slobin, 1994; Slobin, 1996; Hickmann, 2012). La question que nous nous adressons ici est la suivante: que se passe-t-il lorsqu'on acquiert une deuxième langue? En fonction de la L1, le rapprochement vers la LC implique non seulement l'acquisition de nouvelles structures linguistiques, mais aussi une reconceptualisation des informations spatiales à exprimer et de leur distribution dans l'énoncé. En l'état actuel, les travaux relatifs aux situations de déplacement en L2 (voir section 2) indiquent que ce type de restructuration, bien que possible, semble être très difficile à atteindre: les productions en L2, même aux stades très avancés, montrent des divergences par rapport aux choix préférentiels des locuteurs natifs de la LC qui sont souvent attribuées à l'influence de leur L1 (voir Han et Cadierno, 2010; Benazzo et al., 2012b; Bartning, 2009, entre autres).

Partant de ces prémisses, notre étude analyse l'expression des situations de déplacement dans le discours oral d'apprenants de deux langues romanes - le français et l'italien L2 - dans le but d'investiguer:

1. dans quelle mesure les productions en L2 montrent une trace de la L1 et/ou une (re)conceptualisation conforme à la LC lors de l'expression du déplacement en L2;

2. les parcours évolutifs des apprenants, à savoir si et à quel niveau de compétence ils s'approprient les patrons de lexicalisation de la LC.

Pour chaque LC nous avons comparé les productions de sujets dont la L1 est typologiquement proche (italophones en français L2 et francophones en italien L2) vs éloignée (anglophones en italien ou en français L2), représentant deux niveaux de maîtrise de la L2 (intermédiaire et avancé). Le croisement LS-LC choisi vise ainsi à déterminer l'étendue de l'influence translinguistique en fonction des caractéristiques typologiques des langues en contact (langue à cadrage verbal $v$ satellitaire) et son évolution sur la base du niveau atteint en L2.

Dans les sections suivantes, nous allons d'abord présenter les études antérieures sur l'expression du déplacement en L2 (section 2), avant d'exposer la méthodologie 
adoptée pour le recueil et l'analyse de données (section 3). Les résultats de notre étude (section 4) sont répartis en deux sections - la première concerne les productions natives dans les trois langues en contact et la deuxième les productions en L2 - suivie par la discussion des résultats (section 5).

\section{L'expression du déplacement en L2}

Lors de l'appropriation d'une L2, la tâche de l'apprenant consiste à identifier les aspects relatifs aux situations de déplacement à exprimer en LC, qui souvent diffèrent de ceux de la langue source (LS). Le sujet apprenant doit donc apprendre à «repenser pour parler» en s'orientant vers les notions spatiales encodées dans les outils lexicogrammaticaux de la L2 (Cadierno, 2017).

Les similarités et/ou les différences entre les langues en contact peuvent entrấner divers phénomènes d'influence de la L1 dans le discours en L2 (voir Jarvis et Pavlenko, 2010, pour une vue d'ensemble). Il est toutefois utile de souligner que l'influence translinguistique n'a pas lieu de manière mécanique sur la base des différences entre les langues en contact telles qu'attestées par les linguistes ou typologues. La transférabilité des structures peut dépendre d'autres facteurs. En premier lieu, il est nécessaire de prendre en compte (a) l'évaluation «subjective» de l'apprenant (voir psychotypologie, Kellerman, 1979) sur le degré de proximité entre LS et LC, évaluation qui peut évoluer en fonction de son niveau de maitrise: il est plus probable que l'apprenant transfère une construction de sa L1 lorsqu'il juge la LC comme proche de sa langue L1 et si la construction en question est ressentie comme non idiomatique. Ensuite, (b) la fréquence et le caractère typologiquement marqué/non marqué de la structure à apprendre jouent également un rôle important (Eckman, 1996), les constructions plus fréquentes et non marquées étant considérées comme plus transférables. Un autre facteur influent est constitué par (c) le niveau linguistique observé, les phénomènes de transfert affectant de manière différenciée le niveau phonologique, morphosyntaxique, lexical, pragmatique, etc. Finalement, la transférabilité des structures est également influencée par (d) le stade atteint par l'apprenant en L2, puisqu'il est nécessaire de maîtriser des outils linguistiques complexes en L2 afin de pouvoir traduire une structure de la L1 de manière équivalente en L2 (Stutterheim, 2003). Par ailleurs, on a tendance à surestimer le rôle des différences interlinguistiques, alors que les ressemblances semblent avoir un impact plus important sur les phénomènes de transfert. En effet, selon Ringbom et Jarvis (2009), les apprenants seraient plus à la recherche de similarités que de différences entre ce qu'ils connaissent déjà (leur L1) et ce qui est à apprendre (la L2) : «learners are constantly looking for similarities (when they can find them) rather than for differences» (Ringbom et Jarvis, 2009: 106).

Dans ce qui suit, nous reprenons les résultats d'études antérieures qui se sont penchées spécifiquement sur l'encodage des situations de déplacement en L2 et sur les différents types de difficultés rencontrées par l'apprenant d'une L2 tout au long du processus d'apprentissage. 
Les recherches portant sur les stades initiaux de l'acquisition L2 (public de migrants, données longitudinales du projet $\mathrm{ESF}^{3}$ ) indiquent que les apprenants adultes en immersion se forgent assez rapidement des moyens minimaux pour faire référence à l'espace. Au niveau de la «Variété de Base» (voir Klein et Perdue, 1997) - stade caractérisé par l'organisation de l'énoncé autour d'un verbe qui n'est pas encore fonctionnellement fléchi - le lecte de l'apprenant inclut déjà «des moyens linguistiques permettant la localisation d'un objet, l'expression explicite du déplacement et de la direction qu'emprunte l'objet en mouvement. Ces moyens sont limités, et imposent des ordres de constituants dans l'énoncé tels que "source avant but" et "objet avant localisation"» (Perdue, 1993b: 15). Par ailleurs, face à la distinction entre langue à cadrage verbal $v$ s satellitaire, les apprenants semblent opter pour les schémas de la LC: il s'agit notamment de verbes fusionnant déplacement et trajectoire en français (la seule LC romane du projet ESF) tels que arriver/partir, entrer/sortir ou monter/descendre, et de particules directionnelles dans les langues germaniques (telles que up ou away en anglais ou auf/weg en allemand), bien que ces moyens soient souvent utilisés de manière idiosyncrasique ${ }^{4}$. À ce stade, le choix des moyens pour référer à l'espace en L2 montre peu d'influence directe de la LS (voir Becker et Carroll, 1997: 191) tout comme les principes généraux qui régissent cette variété pour l'organisation de l'énoncé (voir Klein et Perdue, 1997), ou l'expression de la temporalité (Dietrich et al., 1995). C'est au niveau des stades intermédiaires ou avancés, avec l'élargissement du répertoire linguistique en L2, que les spécificités de langues en contact (LS et LC) jouent un rôle plus important dans les productions des apprenants. Dans ce qui suit, nous faisons référence à plusieurs études concernant justement des apprenants de ces deux niveaux (intermédiaire/avancé) en considérant principalement des langues cibles romanes, étant donné que notre analyse porte sur le français et l'italien L2.

Les études de Cadierno (2004) et Cadierno et Ruiz (2006), comparant les productions en espagnol L2 (récits de la Frog Story) d'apprenants de L1 danois $v s$ italien, montrent ainsi la présence de stratégies de lexicalisation typiques de la L1 surtout au niveau intermédiaire («satellization of locative constructions», Cadierno et Ruiz, 2006: 189). Les apprenants de niveau avancé, en revanche, arrivent à s'approprier la structure à cadrage typique de la L2 - et donc à repenser pour parler en L2 -, mais pas pour toutes les composantes du mouvement. Pour ce niveau les auteurs constatent, en effet, l'adoption des principes de la LC dans l'expression de la manière mais pas dans celle de la trajectoire, qui s'avère être beaucoup plus détaillée, notamment dans les productions des danois («el niño fue arriba de un árbor "the

3. Le projet «Second Language Acquisition by Adult Immigrants», plus souvent nommé projet ESF puisque financé par la Fondation européenne de la science (European Science Foundation en anglais), a observé de manière longitudinale des migrants dans 5 pays européens, représentant 10 combinaisons de LS-LC (voir Perdue, 1993a).

4. L'extrait suivant illustre le type d'emplois idiosyncrasiques recensés chez un apprenant italophone de l'anglais. En réponse à la question du locuteur natif «What's be doing?», l'apprenant produit «Up the ladder» pour exprimer l'action de monter l'échelle (Becker et Carroll, 1997: 51). 
child went up of a tree"», Cadierno et Ruiz, 2006: 204 [trad. fr. «l'enfant est allé en haut d'un arbre»]), que dans les productions des hispanophones natifs. Sur ce point les chercheurs s'attendaient à des différences entre les apprenants danois et italiens, en raison de la typologie satellitaire du danois L1, alors que les résultats de leur analyse ne permettent pas d'étayer cette hypothèse.

Dans une étude sur l'expression du mouvement provoqué en français L2 (tâche «Popi» ${ }^{5}$ ), Hendriks et al. (2008) soulignent la tendance des apprenants anglais débutants à transférer la distribution des composantes sémantiques de leur L1 en L2: ils expriment la cause et la manière dans la racine verbale, alors que la trajectoire est encodée dans des propositions séparées (ex. "Popi tire/-tiré [Manière + Cause] *une sac /et *ascende [Trajectoire] le toit", au lieu de Popi monte sur le toit en tirant un sac, Hendriks et al., 2008: 30). Les apprenants avancés se rapprochent globalement des structures typiques du français sans pour autant se débarrasser complètement du schéma de lexicalisation de leur L1, étant donné que ce genre de constructions est encore visible dans leurs productions ( $«$ il le tire [Cause + Manière dans le verbe] après lui en traversant la rue [Trajectoire dans un gérondif]», Hendriks et al., 2008: 30). Ces exemples illustrent les effets d'un transfert conceptuel, à savoir le transfert de structures traduisant une perspective discursive typique de la L1 (Jarvis et Pavlenko, 2010).

Hendriks et Hickmann (2011) se sont servies des mêmes apprenants pour une étude sur le mouvement volontaire (description de saynètes animées): les auteurs constatent que les apprenants en question recourent davantage aux schémas typiques de leur L1 face à une tâche plus complexe demandant l'expression de la cause du déplacement. A contrario, pour l'expression du mouvement volontaire, les apprenants semblent adhérer aux patrons du français LC. Autrement dit, quand l'information à encoder est plus complexe, l'apprenant s'appuie sur la façon la plus familière d'organiser une information donnée. De ce fait, la tâche semble également jouer un rôle crucial sur les choix linguistiques des locuteurs et sur le degré de transférabilité de l'organisation informationnelle.

Spreafico et Valentini (2009) et Bernini et al. (2006) ont étudié des apprenants de différentes $\mathrm{LS}^{6}$ en italien L2. Les résultats de leurs études indiquent que ce sont surtout les productions des apprenants anglophones, à tous les niveaux considérés, qui révèlent une plus forte influence de la L1. Ceux-ci utilisent parfois transitivement des verbes de déplacement tels que salire [monter] qui sont intransitifs dans la LC (*il bambino ba salito un albero [l'enfant a monté un arbre] au lieu de il bambino è salito sull'albero): ces emplois peuvent être attribués à l'influence de leur L1 (the child climbed the tree), étant donné l'absence de constructions similaires

5. La tâche «Popi» sollicite l'expression du mouvement volontaire et provoqué. Elle se compose de 32 dessins animés où l'agent, dénommé «Popi», se déplace dans l'espace en provoquant également le déplacement d'un objet. L'agent et le patient suivent la même trajectoire.

6. Les LS des apprenants observés par Spreafico et Valentini (2009) sont l'arabe (variété marocaine et tunisienne), l'albanais, le français, l'anglais, le néerlandais et l'allemand. Bernini et al. (2006) se sont, en revanche, penchés sur les productions d'apprenants allemands, néerlandais et anglais. 
dans les productions d'apprenants ayant comme L1 une autre langue germanique (allemand ou néerlandais). Pour ce qui est du locus d'encodage de la trajectoire, les apprenants allemands et néerlandais semblent exprimer cette composante davantage dans l'élément extraverbal que les anglophones: ce décalage est expliqué par les caractéristiques de l'anglais L1 qui, contrairement aux autres langues germaniques, dispose de deux types de stratégies (go out vs exit) et rend ainsi plus accessibles les constructions à cadrage verbal de la LC.

Dans le cas inverse, à savoir pour les sujets apprenant une langue à cadrage satellitaire, Carroll et al. (2012) ont montré que des apprenants français avancés de l'anglais et de l'allemand L2 n'adoptent que partiellement les structures de déplacement typiquement utilisées par les natifs des deux langues cibles. Plus précisément, ils n'expriment pas les concepts référant aux caractéristiques de l'espace traversé (contours du fond) de manière conforme aux langues à cadrage satellitaires (en anglais, around, along, etc.) et préfèrent plutôt se servir de locatifs: ils produisent ainsi $A$ car is driving on a road au lieu de $A$ car is driving along a road (Carroll et al., 2012: 220), ce qui reflète les usages natifs de leur L1 et, par conséquent, la présence d'un transfert conceptuel. Dans le même sens, Sharpen (2016) a constaté l'influence de la L1 auprès d'apprenants avancés anglophones de l'espagnol L2 et hispanophones de l'anglais L2, cette fois-ci dans une tâche de traduction. Pour le premier groupe, une transposition des structures satellitaires est attestée dans leurs productions, alors que le second groupe d'apprenants a suivi les schémas de lexicalisation spatiale typique des langues à cadrage verbal.

La comparaison de ces résultats n'est pas toujours évidente, étant donné la diversité de tâches employées et sans doute des critères pour déterminer le niveau de maîtrise de la L2. Cependant nous pouvons conclure, comme Cadierno (2017), que les études sur l'expression du déplacement en L2 témoignent d'un transfert conceptuel plus ou moins important dans le discours oral en L2: (a) en fonction du schéma de lexicalisation typique de la L1 (à cadrage verbal ou à cadrage satellitaire), (b) même à des niveaux très avancés, et (c) indépendamment du degré variable de similarité, du point de vue de l'expression du déplacement, entre les langues (res)sources (LS).

Les études mentionnées montrent globalement que les productions en L2 présentent des divergences par rapport aux préférences natives en LC à parité de tâche. Il est toutefois moins clair dans quelle mesure ces écarts reflètent l'empreinte de la L1, ou bien s'ils révèlent des tendances propres aux variétés d'apprentissage d'une LC donnée, incluant des processus de restructuration partielle en fonction du niveau atteint en L2.

\section{Méthode et données}

Notre étude analyse les préférences natives et non natives dans l'expression du déplacement sur la base de données narratives orales semi-spontanées obtenues à partir d'un même support pour tous les participants enregistrés. Le support utilisé 
est l'histoire Frog, Where Are You? (Mayer, 1969), qui a été largement employée dans les recherches en acquisition des langues dans les dernières décennies (voir, entre autres, Berman et Slobin, 1994; Cadierno, 2004). Cette histoire se développe sur 24 images sans texte illustrant un garçon et son chien à la recherche d'une grenouille qui s'est échappée pendant la nuit: elle contient ainsi de nombreux évènements impliquant le déplacement dans l'espace de différents personnages (l'enfant, le chien, la grenouille, un hibou, un cerf, etc.). Chaque sujet a été enregistré individuellement: il a été invité à regarder les images et à en raconter l'histoire à une tierce personne n'ayant pas accès au support, sans aucune contrainte de temps.

Avant d'étudier les productions orales non natives, nous avons analysé les productions en L1 afin d'identifier: a) comment les locuteurs natifs des trois langues ciblées expriment les relations spatiales dynamiques dans la tâche narrative demandée, ainsi que b) le parcours acquisitionnel des apprenants en fonction de leur L1. Nous avons ainsi recueilli des récits oraux auprès de 30 locuteurs natifs adultes (10 sujets par langue: anglais, français et italien) et ensuite des récits auprès d'apprenants qui représentent quatre combinaisons de LS-LC (typologiquement proches $v$ s éloignées) $^{7}: 40$ apprenants de l'italien L2 dont la L1 est soit l'anglais soit le français, et 40 apprenants du français L2, dont la L1 est soit l'anglais soit l'italien (voir tableau 1 pour une vue d'ensemble).

\begin{tabular}{|c|c|c|c|}
\hline L2 & L1 & Niveau & Sujets \\
\hline & Anglais & \multirow{3}{*}{$\mathrm{LN}$} & 10 \\
\hline & Français & & 10 \\
\hline & Italien & & 10 \\
\hline \multirow{4}{*}{ Italien } & \multirow{2}{*}{ Anglais } & Intermédiaire & 10 \\
\hline & & Avancé & 10 \\
\hline & \multirow{2}{*}{ Français } & Intermédiaire & 10 \\
\hline & & Avancé & 10 \\
\hline \multirow{4}{*}{ Français } & \multirow{2}{*}{ Anglais } & Intermédiaire & 10 \\
\hline & & Avancé & 10 \\
\hline & \multirow{2}{*}{ Italien } & Intermédiaire & 10 \\
\hline & & Avancé & 10 \\
\hline & & & 110 \\
\hline
\end{tabular}

Tableau 1 - Les informateurs

7. Dans ce contexte, les langues étudiées sont considérées comme typologiquement proches ou distantes uniquement du point de vue de l'expression du déplacement (locus d'encodage des composantes spatiales). 
En ce qui concerne les productions en L2, les participants sont des étudiants apprenant la LC dans leur pays natal, notamment à l'université College Cork pour les apprenants anglophones, à l'université d'Avignon pour les francophones et à l'université de Naples Federico II pour les italophones. Certains des apprenants enregistrés (notamment 14 participants sur 80 ) ont également eu la possibilité de faire un séjour d'études Erasmus dans le pays de leur L2. Ils ont tous commencé à apprendre la LC à l'âge adulte et connaissaient au moins une autre langue étrangère avant leur participation à l'étude. Pour chaque combinaison de langues, deux niveaux de compétence en LC ont été étudiés: intermédiaire vs avancé. Les niveaux ont été établis essentiellement en fonction du degré de complexité morpho-syntaxique (Bartning et Schlyter, 2004) identifié dans les productions orales recueillies en LC. Plus spécifiquement, le niveau intermédiaire englobe les apprenants dont les productions présentent des distinctions très fragiles forme-fonction dans le domaine de la morphologie verbale (par exemple, utilisation de l'infinitif dans un contexte fini, emploi inapproprié du passé composé/imparfait) et privilégient la parataxe. Les apprenants avancés, par contre, recourent à une morphologie verbale plus diversifiée (par ex., emploi du subjonctif ou du passé simple), utilisée dans la plupart des cas à bon escient, et construisent leur discours à travers des énoncés multipropositionnels. La répartition des apprenants selon leur niveau de maitrise tient donc compte de la connaissance de la LC lors du recueil des données et non du nombre d'années d'études précédentes.

Les 110 productions recueillies ont été transcrites selon les conventions CHAT (Codes for the Human Analysis of Transcripts, MacWhinney, 2000). Toute proposition (clause) contenant une référence à un évènement de déplacement a été codée et analysée selon des paramètres inter-reliés en procédant de la manière suivante:

1. identification du type d'information spatiale exprimée (Trajectoire, Manière, Cause) tous moyens confondus et de son locus, à savoir distribution de la composante exprimée entre la racine verbale et les éléments extraverbaux;

2. détermination du rapport types/tokens des verbes de déplacement ${ }^{8}$. À ce propos, les catégories verbales observées incluent: les verbes de trajectoire (ang. fall, fr. tomber, it. cadere), les verbes neutres (ang. go, fr. aller, it. andare), les verbes de manière (ang. run, fr. courir, it. correre), les verbes de cause (ang. put, fr. mettre, it. mettere), les verbes de trajectoire et de manière (ang. dive, fr. plonger, it. tuffarsi), les verbes de manière et de cause (ang. throw, fr. lancer, it. lanciare) et les verbes de cause et de trajectoire (ang. let fall, fr. faire tomber, it. far cadere);

8. L'identification des verbes de déplacement et leur classement dans différentes catégories (trajectoire, manière, cause, etc.) est une tâche assez complexe pour les chercheurs. Cette tâche est liée essentiellement aux différents aspects du déplacement que les chercheurs veulent investiguer, d'où la présence dans la littérature de diverses catégorisations des verbes de déplacement. Cette étude s'est inspirée des critères de classement suivis par Berman et Slobin (1994), Hickmann et al. (2014), et en partie Spreafico (2009). 
3. analyse de la nature syntaxique des moyens extraverbaux utilisés (locutions adverbiales, particules, syntagmes prépositionnels [SP]) et des notions sémantiques qu'ils encodent. Par ailleurs, nous avons également considéré le degré d'empaquetage des informations portant sur la trajectoire (notion d'event conflation, voir Berman et Slobin, 1994), notamment dans quelle mesure les locuteurs expriment aussi bien la source que le but dans la même proposition (ex. ang. the dog has managed to get the beebive down from the tree [source] onto the ground [but]) au lieu de les encoder dans des propositions distinctes (ex. fr. le chien fit tomber le nid de l'arbre [source] // le nid était tombé par terre [but]).

La méthodologie adoptée pour cette étude prévoit des comparaisons croisées entre, d'une part, les récits des groupes natifs et non natifs et, d'autre part, entre les différents groupes d'apprenants. Afin d'évaluer si les distributions des fréquences diffèrent en fonction de la L1 ou du niveau atteint en L2, nous avons utilisé le test chi-carré de Pearson $\left(\chi^{2}\right)^{9}$ pour estimer l'homogénéité des groupes. Pour ce faire, nous avons saisi le groupe et le type de phénomène observé comme variables indépendantes et les occurrences brutes (nombre de fréquences) comme variables dépendantes dans un tableau de contingence.

\section{Analyse des données et résultats}

\subsection{Perspectives préférentielles en $\mathrm{L} 1$}

Globalement, les locuteurs natifs de l'anglais, du français et de l'italien préfèrent porter leur attention massivement sur l'expression de la trajectoire tous moyens confondus (voir figure 1 ci-après). Néanmoins, une différence statistiquement significative caractérise le choix et la distribution des moyens linguistiques dans les trois groupes en question concernant l'encodage de la trajectoire, de la manière et de la cause du déplacement $\left(\chi^{2}(4)=13,69, p<0,05\right)$. Plus précisément, la manière est plus fréquemment exprimée par les anglophones (run, jump, spike, tumble, creep, sneak, pop, tiptoe, climb, etc.; voir tableau 2), ce qui confirmerait le statut de l'anglais comme langue bigh-manner salient par rapport au français et à l'italien (Slobin, 2004 et 2006; Cardini, 2008).

\begin{tabular}{|l|r|r|r|r|}
\hline & ANG & \multicolumn{1}{|l|}{ FRA } & ITA & \multicolumn{2}{|l|}{$\boldsymbol{\chi}^{2}$ test } \\
\hline Trajectoire & 342 & 262 & 236 & 0,09 \\
\hline Manière & 82 & 37 & 30 & $\mathbf{0 , 0 0 3}^{*}$ \\
\hline Cause & 40 & 42 & 32 & 0,22 \\
\hline
\end{tabular}

Tableau 2 - Composantes spatiales chez les locuteurs natifs

9. Nous rappelons la formule du test chi-carré: $\chi^{2}$ (degré de liberté) $=$ chi-square, $p<0,05$. 


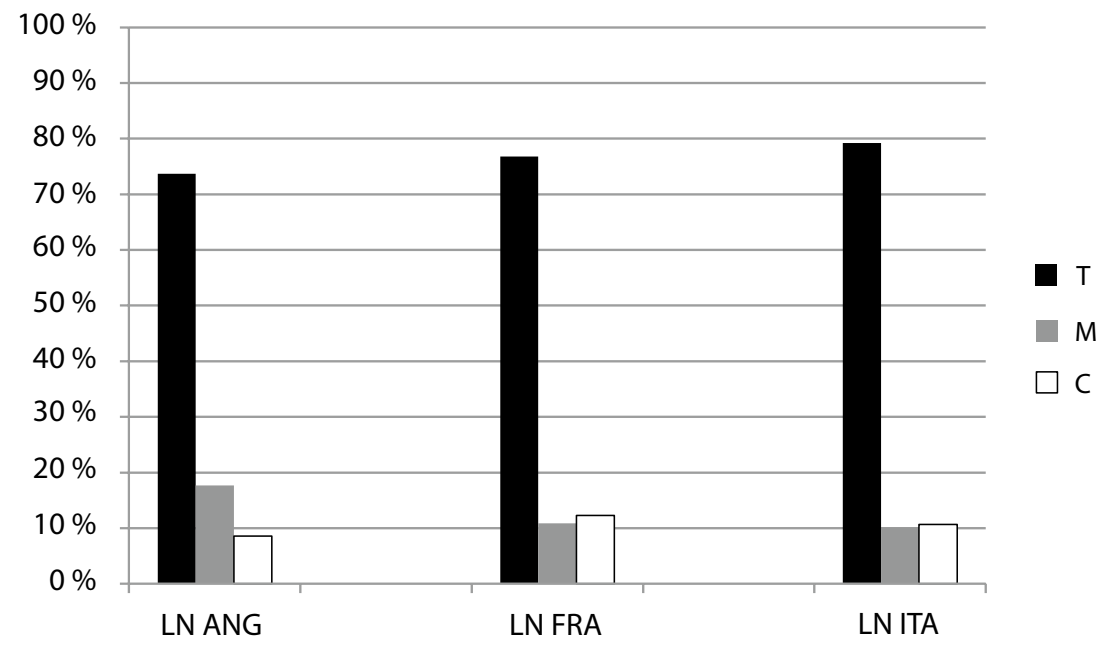

Figure 1 - Informations spatiales exprimées par les locuteurs natifs des trois langues ( $\mathrm{T}=$ Trajectoire $\mathrm{M}=$ Manière $; \mathrm{C}=$ Cause $)$

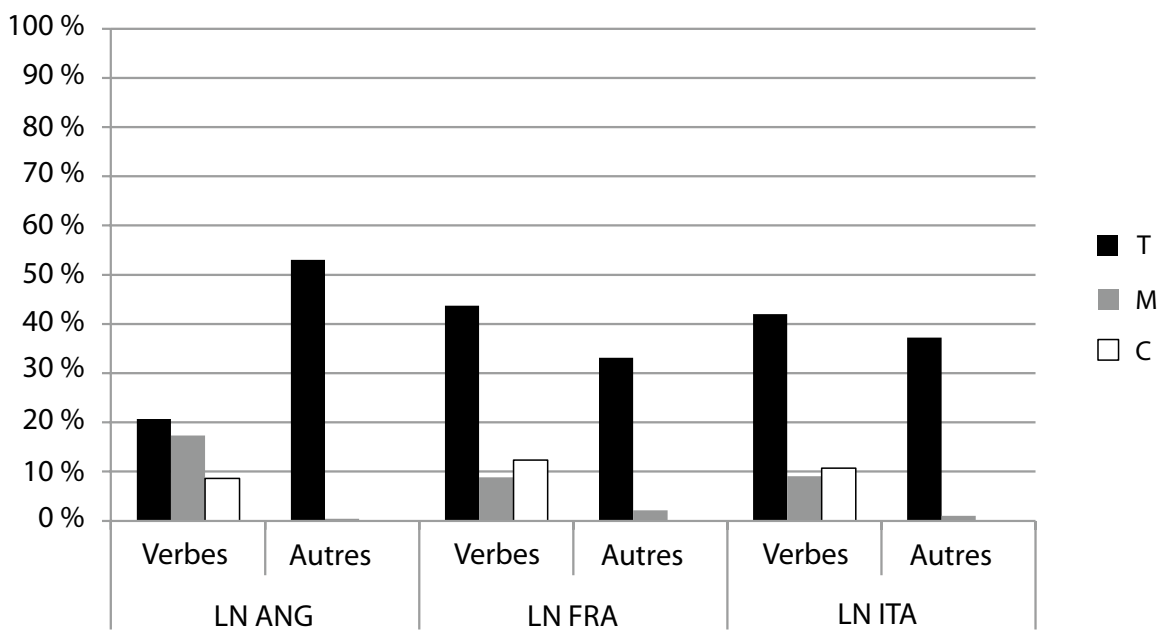

Figure 2-Locus de l'information spatiale chez les locuteurs natifs

La figure 2 visualise le locus des informations spatiales, à savoir la distribution de l'information entre la racine verbale et les éléments autres que le verbe (particules, locutions adverbiales et SP) dans les trois langues.

À ce propos, les anglophones représentent le seul groupe qui tend à exprimer la trajectoire plus à l'extérieur du verbe $(53 \%)$ que dans la racine verbale $(20,7 \%)$, ce qui les oppose aux locuteurs des deux langues romanes qui préfèrent le schéma 
inverse. En effet, les francophones et les italophones présentent des pourcentages relativement similaires: la trajectoire est lexicalisée dans la racine verbale dans $43,7 \%$ des cas chez les premiers et dans $42 \%$ des cas chez les derniers, bien que les italophones se servent légèrement davantage de moyens extraverbaux que les francophones $(37,2 \%$ vs $33,1 \%)$.

Les locuteurs de deux langues romanes en question diffèrent plutôt dans le type de moyens extraverbaux utilisés pour l'encodage de la trajectoire (voir tableau 3 ci-après). Nous constatons l'exploitation de particules extraverbales $(13,2 \%)$ dans les productions des italophones, tendance non attestée dans nos données natives francophones.

L'emploi des particules dans des constructions satellitaires en italien se fait par le biais des VS (andare via [aller au loin = s'en aller], saltare via [sauter au loin], uscire fuori [sortir dehors] ; voir Anastasio, 2017 et 2018; Cini, 2008; Iacobini et Masini, 2007), jamais attestées dans les récits de nos locuteurs francophones. En résumé, les locuteurs des deux langues romanes en question ne présentent pas d'écart important entre le pourcentage d'emploi des verbes de trajectoire et celui des moyens extraverbaux encodant cette information spatiale: ils se comportent de la même manière, mais à travers l'exploitation de moyens linguistiques partiellement différents (particules pour les italophones, adverbiaux pour les francophones et/ou SP pour les deux groupes).

La variation des moyens attestés en italien pour l'encodage de la trajectoire permet de considérer l'italien comme une langue à baute saillance de trajectoire - malgré son statut de langue à dominante cadrage verbal - en raison de l'emploi des VS, qui sont distribués de manière homogène chez tous les natifs observés.

Parmi les moyens autres que les verbes, les SP indiquant l'une des phases de la trajectoire (source et/ou but) ont été largement produits par tous les sujets. L'intérêt d'étudier les SP dans les récits réside dans notre désir de voir si, comme le montrent Berman et Slobin (1994), les locuteurs des langues satellitaires ont tendance à compacter la source et le but du déplacement dans la même proposition, alors que les locuteurs des langues à cadrage verbal y arriveraient très rarement, car ils ont besoin de constructions plus complexes pour ce faire. De notre analyse il ressort que les anglophones peuvent effectivement exprimer la source et le but du déplacement dans la même proposition (he lets the boy fall over the cliff into the river with his dog), mais ils ne le font que très rarement ( 5 occurrences de ce type sur 118 situations de déplacement où apparaissent des SP vs aucune occurrence en français et en italien). Dans la plupart des cas, ils encodent plutôt les deux informations dans des propositions séparées, tout comme le font les francophones et les italophones (ex. fr. le cerf a fait tomber le garçon et le chien du précipice // et ils sont tombés dans le lac; ex. it. caddero da questo dirupo // e caddero in uno stagno). Ainsi, contrairement à ce qui a été attesté dans les études antérieures, nos données révèlent une certaine liberté chez les locuteurs anglophones quant au choix d'empaqueter plusieurs informations au sein de la même proposition ou dans plusieurs propositions indépendantes. 


\begin{tabular}{|l|r|r|}
\hline & FRA & ITA \\
\hline SP & $90,8 \%$ & $84,2 \%$ \\
\hline Particules & $0 \%$ & $13,2 \%$ \\
\hline Locutions adverbiales & $9,2 \%$ & $2,6 \%$ \\
\hline
\end{tabular}

Tableau 3 - Éléments extraverbaux utilisés dans les récits natifs en français et en italien

\begin{tabular}{|l|l|r|r|r|r|r|r|r|}
\hline & $\begin{array}{l}\text { Verbes de } \\
\text { déplacement/ } \\
\mathbf{n}^{\circ} \text { proposition }\end{array}$ & Neutre & T & M & T + M & C & C + M & C + T \\
\hline $\begin{array}{l}\text { LN } \\
\text { ANG }\end{array}$ & $211 / 771(27,3 \%)$ & 33 & 65 & 53 & 20 & 22 & 7 & 11 \\
\hline $\begin{array}{l}\text { LN } \\
\text { FRA }\end{array}$ & $191 / 580(33 \%)$ & 9 & 113 & 13 & 14 & 17 & 3 & 22 \\
\hline $\begin{array}{l}\text { LN } \\
\text { ITA }\end{array}$ & $178 / 599(29,8 \%)$ & 12 & 111 & 16 & 7 & 21 & 4 & 7 \\
\hline
\end{tabular}

Tableau 4 - Verbes de déplacement utilisés par les locuteurs natifs (occurrences brutes)

En ce qui concerne les verbes de déplacement, nous avons considéré le rapport types/tokens de ceux-ci afin de vérifier si les locuteurs des langues à cadrage satellitaire produisent plus de verbes de déplacement que ceux des langues à cadrage verbal (Berman et Slobin, 1994). Dans les données natives analysées, les anglophones utilisent 40 types de verbes différents, les francophones 39 et les italophones 35 . Cependant, le nombre inégal de tokens ainsi que de propositions totales des trois groupes, nous empêche de tirer des conclusions précises sur ce point, si ce n'est qu'il y a une correspondance entre ces diverses unités de mesure : le groupe utilisant plus de propositions produit également plus de verbes de déplacement (tableau 4) et davantage de types différents. La véritable différence réside plutôt dans les types de verbes de déplacement exploités et dans leur distribution chez les informateurs. À titre d'exemple, en anglais L1 les verbes de trajectoire ainsi que les verbes de manière (types de verbes majoritairement exprimés) sont distribués de façon homogène chez tous les locuteurs. En revanche, cette homogénéité se retrouve en français uniquement pour les verbes de trajectoire, dont tous les locuteurs se sont servis, alors que les autres types de verbes (manière et/ou cause) sont employés très peu et n'apparaissent pas dans toutes les productions. De même, pour l'italien L1, ce sont toujours les verbes de trajectoire qui apparaissent largement dans tous les récits, alors que les catégories verbales de manière et/ou cause n'apparaissent ni fréquemment ni dans toutes les productions. 
Si on part du présupposé que le système linguistique et conceptuel de la L1 influence fortement les productions en L2, à la lumière des tendances relevées auprès des natifs on peut s'attendre à ce que les apprenants anglophones (a) expriment davantage la manière du déplacement en LC (notamment dans la racine verbale) par rapport aux apprenants de L1 romane, et (b) qu'ils encodent la trajectoire en dehors du verbe. Cette dernière tendance des anglophones pourrait être partiellement partagée avec les apprenants italophones en français L2, étant donné que l'italien présente également l'option à cadrage satellitaire à travers l'exploitation de verbes à particules apparentés à ceux de l'anglais ${ }^{10}$.

\subsection{Les productions en $\mathrm{L} 2$}

Comme nous l'avons également vu pour les groupes de contrôle, tous les apprenants à tout niveau ont massivement encodé la composante trajectoire (voir figures $3 \mathrm{a}-\mathrm{b}$ ci-après). Cette tendance commune, partagée par tous les groupes d'apprenants, est probablement attribuable au fait que l'expression de la trajectoire représente la composante essentielle dans l'expression de ce type de situation communicationnelle (Talmy, 1985).

En ce qui concerne les autres composantes, la manière est légèrement plus encodée que la cause chez les apprenants anglophones, alors que les apprenants francophones et italophones font précisément l'inverse. Il s'agit toutefois de différences quantitativement minimales et statistiquement non significatives ${ }^{11}$. Tous les groupes adoptent donc un comportement relativement similaire quant à l'encodage des trois composantes spatiales tous moyens confondus.

Pour ce qui est du locus de l'information (voir figures 4a-b ci-après), globalement tous les apprenants ont tendance à exprimer la trajectoire surtout dans la racine verbale, même s'ils recourent également de manière importante à des éléments extraverbaux, qui correspondent principalement à des SP directionnels (source, phase médiane, but). La seule exception à cette tendance est observée chez les anglophones avancés de l'italien L2: ces derniers encodent la trajectoire à proportions égales dans le verbe et dans sa périphérie (40,3\% respectivement).

10. Dans ce contexte, nous tenons à souligner que d'après Kopecka (2006) le français aussi présenterait des constructions à cadrage verbal, notamment à l'aide de préverbes (ac-courir, par-courir, ap-porter, em-porter). Ces préfixes, ayant perdu leur productivité, témoignent du résidu de l'ancien français, qui, à l'instar du latin, présentait un système à cadrage satellitaire. Toujours dans une perspective diachronique, Fagard (2019) a montré la quasi-disparition des particules de trajectoire dans le passage du français médiéval au français moderne. En ce qui concerne le français contemporain, il a mis en évidence la perte de productivité et d'autonomie de tels moyens linguistiques contrairement à la forte productivité des particules de l'italien.

11. Le test $\chi^{2}$ confirme l'absence de différences statistiquement significatives pour l'encodage de la manière et de la cause du déplacement (INT = niveau intermédiaire; AVA = niveau avancé $)$ : apprenants anglophones INT $v$ s AVA Fr. L2: $\chi^{2}(1)=1,9, p>0,05$; apprenants italophones INT $v$ s AVA Fr. L2: $\chi^{2}(1)=0,05, p>0,05$; apprenants anglophones INT $v$ s AVA It. L2: $\chi^{2}(1)=0,14, p>0,05$; apprenants francophones INT $v$ s AVA It. L2: $\chi^{2}(1)=0,10, p>0,05$. 


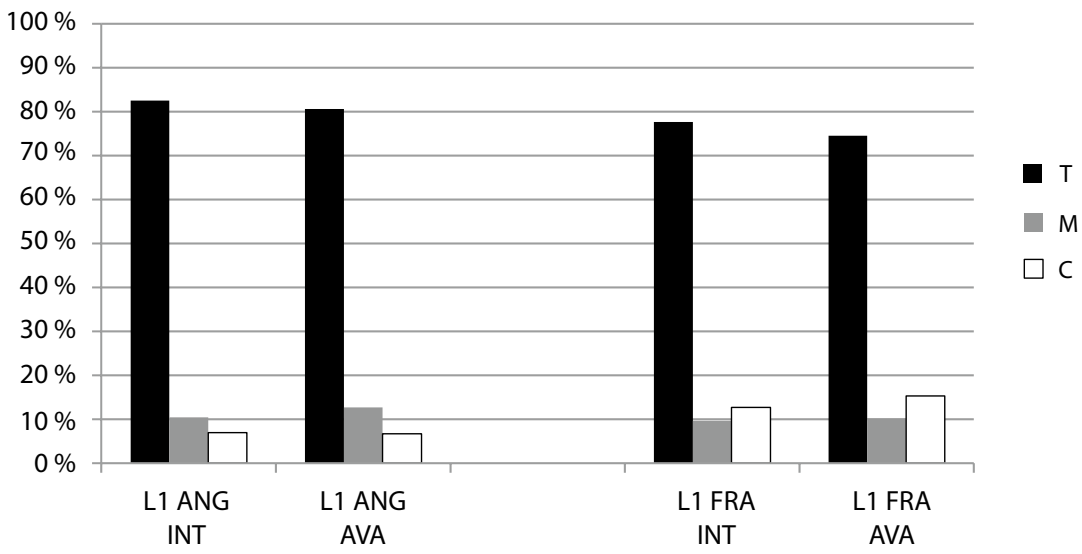

Figure $3 \mathrm{a}$-Composantes spatiales exprimées tous moyens confondus en italien L2 $(\mathrm{INT}=$ niveau intermédiaire $; \mathrm{AVA}=$ niveau avancé $)$

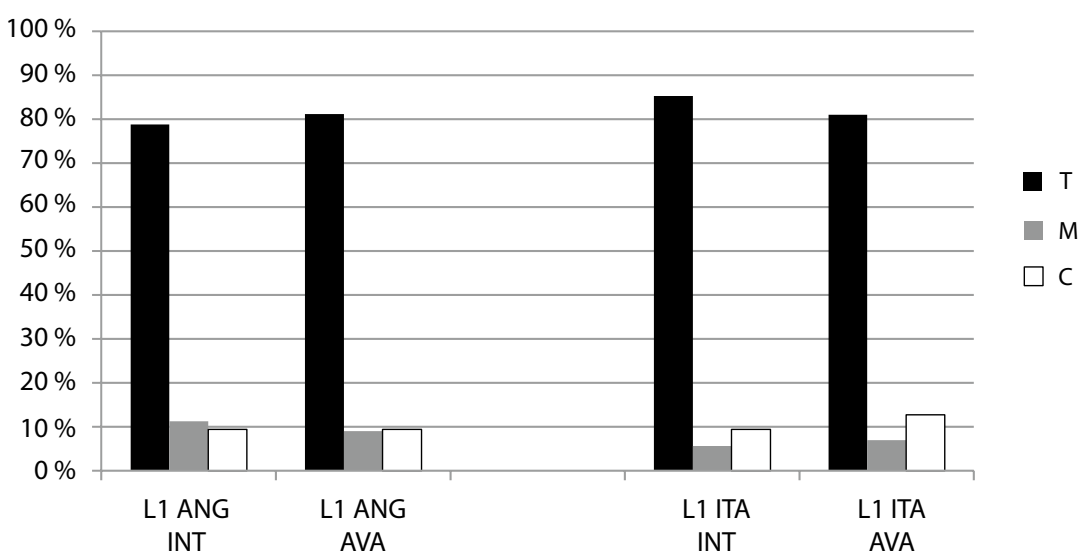

Figure $3 \mathrm{~b}$ - Composantes spatiales exprimées tous moyens confondus en français L2

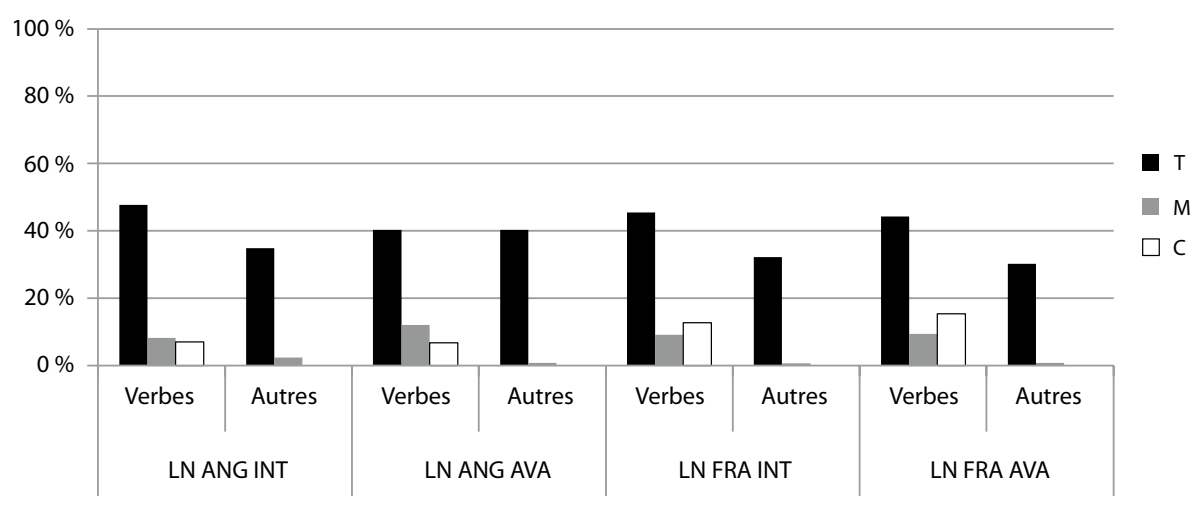

Figure $4 \mathrm{a}-$ Locus de l'information spatiale en italien L2 


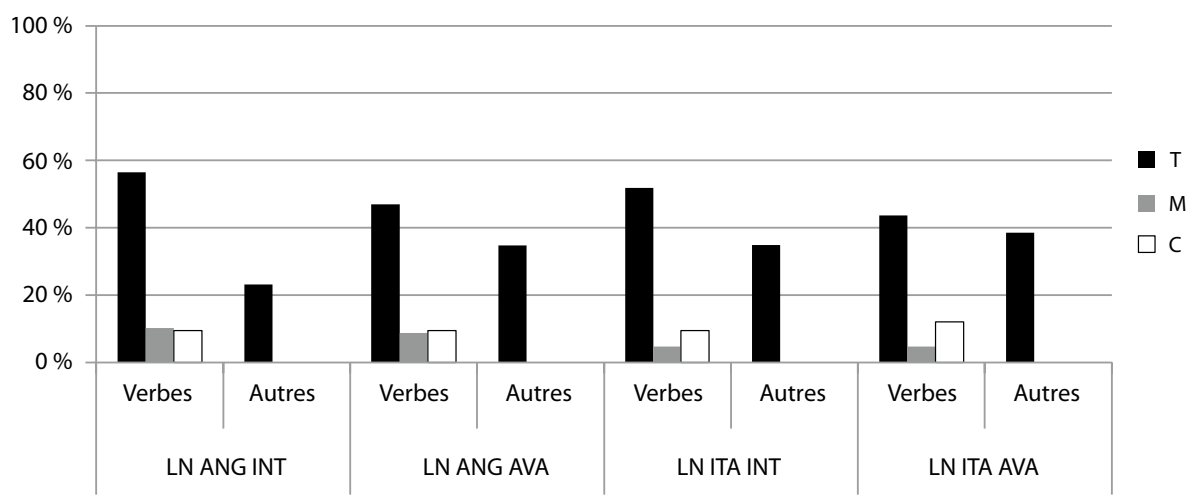

Figure 4b - Locus de l'information spatiale en français L2

Ce qui distingue ce groupe d'apprenants des autres est essentiellement leur emploi assez important de particules directionnelles qui sont intégrées dans les VS italiens (exemples [1a]-[1c]): il s'agit de 25 occurrences, ce qui correspond à $23 \%$ des moyens extraverbaux. Ce type de constructions est en revanche absent chez les intermédiaires (aussi bien anglophones que francophones) de l'italien L2, et assez rare chez les francophones avancés (4 occurrences: andare fuori [aller dehors] [×2], portare via [emporter au loin], cadere fuori [tomber dehors]). Notons également leur absence dans les productions en français L2, quels que soient le niveau et la L1 des apprenants. Ainsi, le test chi-carré nous confirme la présence de différences statistiquement significatives dans la distribution des éléments extraverbaux $\left(\chi^{2}(4)=\right.$ $12,78, p<0,05)$ chez les apprenants.

\section{[1a] poi loro vanno via}

'puis ils [l'enfant et le chien] vont au loin'

[1b] il cane é riuscito a portare giù la casa delle api

'le chien a réussi à porter en bas la maison des abeilles'

[1c] la rana sta andando fuori dal vaso

'la grenouille est en train d'aller hors du bocal'

Les VS sont distribués de manière assez homogène chez les anglophones (ils sont produits par 8 sujets avancés sur 10), ce qui les rapproche des usages natifs de la LC. Par ailleurs, ils ont été exploités aussi bien par les apprenants n'ayant été exposés qu'à l'input institutionnel dans leur pays natal que par ceux ayant également bénéficié d'un séjour d'études en Italie. L'emploi de telles constructions uniquement par les anglophones suggère la présence d'un transfert dû à la similarité structurelle entre les constructions satellitaires de l'anglais et de l'italien. Cette similarité est sans doute favorisée par l'input de l'italien, mais exploitée uniquement au niveau avancé. 
Pour ce qui est du rapport types/tokens, nous nous attendions à ce que les apprenants utilisent moins de types de verbes de déplacement que les locuteurs natifs des variétés cibles, comme il a été constaté dans les études antérieures (voir, par exemple, Cadierno, 2004). Or, aucune différence importante n'a été attestée entre le nombre de verbes de déplacement utilisés chez les deux groupes d'apprenants et les locuteurs natifs (tableau 5 ; nous rappelons les chiffres équivalents attestés pour les locuteurs natifs à la section 4.1:35 types pour les italophones et 39 types pour les français). En revanche, l'une des différences à remarquer est que, comme on pouvait s'y attendre, les apprenants avancés mobilisent un nombre supérieur de types que les intermédiaires (tableau 5 pour les différences inter-niveaux), ce qui va de pair avec l'enrichissement progressif du vocabulaire aux stades avancés d'acquisition. Le fait que cette différence soit moins évidente chez les apprenants francophones et les apprenants italophones pourrait s'expliquer par la transparence lexicale entre le français et l'italien - les récits natifs dans ces deux langues montrent l'emploi de lexèmes verbaux très similaires tels que retourner/ritornare, entrer/entrare, partir/partire -, ce qui faciliterait un emploi plus diversifié de types verbaux chez ces apprenants dès le niveau intermédiaire.

Encore une fois, la catégorie verbale majoritairement exploitée par tous les apprenants et qui contient le plus grand nombre de types est celle des verbes de trajectoire (tableaux 6a-b; voir aussi Cadierno et Ruiz, 2006, entre autres). Les verbes neutres de déplacement ont été retrouvés dans les récits de la plupart des apprenants, alors que les verbes de manière se distribuent de façon très hétérogène chez les apprenants. Les verbes de cause, en revanche ont été utilisés par tous les apprenants francophones avancés. Finalement, les verbes à deux composantes spatiales commencent à devenir plus importants chez les apprenants avancés, ce qui, à nouveau, va de pair avec l'enrichissement lexical à ce stade acquisitionnel. En somme, l'analyse types-tokens nous montre un comportement assez similaire chez nos groupes d'informateurs, à savoir aussi bien chez les apprenants dont la L1 et la L2 partagent le même statut typologique - apprenants italiens du français et vice versa - que chez les apprenants dont la $\mathrm{L} 1$ et la $\mathrm{L} 2$ sont considérées comme typologiquement éloignées - apprenants anglais du français ou de l'italien.

\begin{tabular}{|c|c|}
\hline \multicolumn{2}{|c|}{ Italien L2 } \\
\hline ANG INT & 19 \\
\hline ANG AVA & 33 \\
\hline FRA INT & 31 \\
\hline FRA AVA & 35 \\
\hline \multicolumn{2}{|c|}{ Français L2 } \\
\hline ANG INT & 18 \\
\hline ANG AVA & 37 \\
\hline ITA INT & 24 \\
\hline ITA AVA & 30 \\
\hline
\end{tabular}

Tableau 5 - Types de verbes de déplacement en L2 (occurrences brutes) 


\begin{tabular}{|c|c|c|c|c|}
\hline & \multicolumn{2}{|c|}{ Apprenants anglophones } & \multicolumn{2}{|c|}{ Apprenants francophones } \\
\hline & INT & AVA & INT & AVA \\
\hline Neutre & 15 & 36 & 14 & 15 \\
\hline $\mathrm{T}$ & 39 & 85 & 64 & 81 \\
\hline M & 6 & 21 & 12 & 15 \\
\hline $\mathrm{T}+\mathrm{M}$ & 1 & 9 & 2 & 7 \\
\hline $\mathrm{C}$ & 5 & 9 & 11 & 14 \\
\hline $\mathrm{C}+\mathrm{M}$ & 0 & 0 & 1 & 2 \\
\hline $\mathrm{C}+\mathrm{T}$ & 1 & 8 & 9 & 22 \\
\hline
\end{tabular}

Tableau 6a- Occurrences des types verbes de déplacement en italien L2

\begin{tabular}{|c|c|c|c|c|}
\hline & \multicolumn{2}{|c|}{ Apprenants anglophones } & \multicolumn{2}{|c|}{ Apprenants italophones } \\
\hline & INT & AVA & INT & AVA \\
\hline Neutre & 8 & 7 & 20 & 13 \\
\hline $\mathrm{T}$ & 94 & 108 & 70 & 81 \\
\hline M & 14 & 10 & 7 & 3 \\
\hline$T+M$ & 2 & 13 & 2 & 9 \\
\hline $\mathrm{C}$ & 11 & 16 & 13 & 17 \\
\hline$C+M$ & 3 & 1 & 0 & 1 \\
\hline $\mathrm{C}+\mathrm{T}$ & 3 & 9 & 4 & 10 \\
\hline
\end{tabular}

Tableau 6b - Occurrences des types verbes de déplacement en français L2

Une autre tendance commune, relevée dans les récits des intermédiaires, concerne l'emploi d'emprunts (escape, follow, ascend, pull), de calques ( ${ }^{*}$ scapparsi calqué sur le verbe français s'échapper ou échapper calqué sur les formes verbales anglaise et italienne escape et scappare) et de formes verbales idiosyncrasiques $\left({ }^{*}\right.$ seguetare au lieu de seguire [suivre]), qui témoignent de lacunes lexicales typiques de ce stade concernant les prédicats exprimant le déplacement. Leur présence se raréfie au niveau avancé.

Par ailleurs, certains anglophones (un apprenant avancé et plusieurs apprenants intermédiaires) emploient de manière transitive des verbes qui sont intransitifs dans la LC. Plus précisément, un apprenant anglophone avancé de l'italien L2 a parfois produit la forme idiosyncrasique * montare, calqué sur le verbe français monter, suivi d'un complément d'objet direct (montare l'albero [*monter l'arbre], exemple [2]) dans des contextes où, en revanche, il devrait être suivi d'un SP directionnel $\left({ }^{*}\right.$ montare sull'albero > salire sull'albero [monter sur l'arbre]): 
De même, chez certains apprenants intermédiaires du français nous avons constaté l'emploi transitif du verbe de déplacement monter (exemple [3]) pour décrire des situations de déplacement où les anglophones natifs ont employé le verbe climb suivi d'un complément d'objet direct, tel que climb a tree. Dans les deux cas, il s'agit de transfert syntactico-sémantique, nonobstant le manque de ressemblance formelle entre les deux verbes.

\section{[3] il monte la pierre}

Concernant l'empaquetage des SP directionnels au sein de la même proposition, les apprenants anglophones ont très rarement exprimé la source et le but du déplacement dans la même proposition, que ce soit en italien L2 (2 occurrences chez les intermédiaires et 1 seule occurrence chez un apprenant avancé, voir exemple [4a]) ou en français L2 (4 cas d'event conflation sur 88 propositions contenant des SP directionnels; 1 occurrence chez un sujet avancé, voir exemple [4b]). Tous les groupes d'apprenants préfèrent ainsi encoder les informations directionnelles dans des propositions séparées (voir exemples [4c] et [4d]), tout comme l'ont fait les groupes de contrôle observés.

[4a] il bambino è caduto dalla finestra (source) sulla terra (but) 'l'enfant est tombé de la fenêtre sur le sol'

[4b] le garçon et le chien sont tombés du précipice (source) dans une rivière (but)

[4c] questo cervo ha lanciato il * *egazzo e il suo cane dalla scogliera (source) // il *regazzo è caduto in acqua (but)

'ce cerf a lancé le garçon et son chien de la falaise // le garçon est tombé dans l'eau'

[4d] a un certo punto il bambino cade dalla *falesa (source) con il cane // e tutti e due sono caduti in un lago (but)

à un moment donné l'enfant tombe de la falaise avec le chien // et tous les deux sont tombés dans un lac'

Il semblerait donc que la tâche utilisée laisse aux locuteurs une certaine liberté quant au choix de compacter les informations directionnelles dans une seule proposition ou séparément, ce qui se manifeste également lors de l'acquisition d'une L2 ${ }^{12}$.

12. Nous rappelons, à ce propos, que les apprenants intermédiaires/avancés tendent à la décomposition, c'està-dire à structurer le discours d'une manière plus analytique par rapport aux locuteurs natifs (Watorek, 1996; Bartning, 1997). Toutefois, dans notre cas il semble difficile et hasardeux d'interpréter l'encodage de la source et du but du déplacement dans deux propositions séparées comme une tendance propre à l'apprenant, étant donné que le même phénomène de décomposition a été attesté dans les récits natifs. 


\section{Discussion des résultats et conclusion}

L'objectif principal de ce travail a été d'investiguer l'expression des situations de déplacement dans une tâche narrative orale effectuée par des apprenants adultes de l'italien et du français L2, représentant quatre croisements de LS-LC: typologiquement proches (français $\mathrm{L} 1>$ italien L2 et vice versa) vs éloignées (anglais L1 > italien L2, anglais L1 > français L2). Cette combinaison de langues nous a permis de vérifier l'étendue de l'influence translinguistique et l'évolution de celle-ci en fonction du niveau de compétence en L2, à savoir intermédiaire vs avancé.

Les trois langues considérées dans notre étude - anglais, français et italien présentent des divergences quant au type de composante spatiale exprimée et aux moyens linguistiques pour ce faire. Plus particulièrement, l'anglais laisse la racine verbale libre pour exprimer la notion de manière alors que la trajectoire est essentiellement encodée dans des particules extraverbales. L'italien et le français, en revanche, suivent les schémas de lexicalisation typiques des langues à cadrage verbal: moins d'attention prêtée à la manière et trajectoire lexicalisée dans le verbe. Néanmoins, l'analyse des données natives a bien mis en relief la possibilité en italien d'encoder la trajectoire dans des particules directionnelles (via, fuori), ce qui ne se manifeste pas en français. Ce résultat nous permet de confirmer l'existence d'une variation intra-typologique entre ces deux langues romanes pour l'encodage des informations spatiales.

Les résultats issus de l'analyse des productions non natives ont fait ressortir aussi bien des similarités que des divergences dans les parcours évolutifs des apprenants observés, qui peuvent s'expliquer par un «effet de niveau» (Benazzo et al., 2012a) et/ou par l'influence de la $\mathrm{L} 1$ respectivement.

Une tendance commune à tous les apprenants, bien qu'avec une fréquence plus élevée chez les intermédiaires, réside dans la conceptualisation «neutre» de la tâche, au sens où elle ne reflète pas les spécificités de la L1. Plus particulièrement, les apprenants traitent la tâche de façon minimale (traitement prototypique, Perdue, 1993a et b; Watorek, 1996) en privilégiant l'expression de la trajectoire, élément de base des événements de mouvement se déroulant dans la tâche employée (voir Hendriks et Hickmann, 2011, pour les mêmes résultats), avant de développer les moyens pour encoder également d'autres informations spatiales. La même tâche est traitée de façon plus complexe aux stades avancés grâce à la mise en œuvre de moyens linguistiques véhiculant d'autres informations/perspectives spatiales qui permettent à l'apprenant d'enrichir son récit (par exemple, emploi de verbes lexicalisant deux informations spatiales à la fois: lancer $[\mathrm{C}+\mathrm{T}]$, grimper $[\mathrm{T}+\mathrm{M}])$.

D'autres tendances communes ont été relevées chez tous les apprenants de niveau intermédiaire:

- emploi d'emprunts (escape, ascend), de calques (montare en italien L2, calqué sur le français monter), de formes verbales ("e prova di *ampicare l'albero»: rampicare au lieu de arrampicarsi; trad. fr. «il essaie de grimper 
[sur] l'arbre») et prépositionnelles («*dans le trou/sont/ un hibou»: dans le trou au lieu de $d u$ trou) idiosyncrasiques;

- utilisation d'une gamme de verbes de déplacement assez restreinte par rapport à celle exploitée par les apprenants avancés.

Ces phénomènes sont spécifiques au stade intermédiaire puisqu'ils se raréfient chez tous les apprenants avancés. Avec l'élargissement du répertoire de moyens linguistiques pour référer aux situations de déplacement, il y a en effet très peu de cas de formes idiosyncrasiques et d'emprunts aux stades avancés: ces sujets enrichissent leurs récits en exprimant davantage de composantes sémantiques lexicalisées au sein de la même proposition (par ex. augmentation des verbes à deux composantes). Ils s'approprient donc progressivement les structures typiques de leur variété cible.

Globalement, nous notons très peu d'influence de la L1 pour l'expression du déplacement en L2 au niveau intermédiaire. Celle-ci semble concerner uniquement la présence d'un répertoire plus large de verbes de déplacement chez les apprenants italophones du français et francophones de l'italien par rapport aux anglophones de même niveau, ce qui peut s'expliquer par une majeure transparence lexicale entre les deux langues romanes. Un effet similaire a également été constaté par Cadierno et Ruiz (2006) chez les apprenants italophones en espagnol L2 face aux apprenants danois de la même LC.

La divergence des parcours acquisitionnels concerne surtout les stades avancés. Comme déjà attesté par Bernini et al. (2006) en italien L2, seuls les apprenants anglophones emploient transitivement certains verbes intransitifs de leur variété cible : ils produisent par exemple monter la pierre au lieu de monter sur la pierre pour la description de certaines scènes de la Frog Story où les natifs anglais utilisent le verbe climb suivi d'un complément d'objet direct (climb a tree, climb a rock). Il s'agit d'un transfert syntactico-sémantique qui a lieu nonobstant le manque de ressemblance formelle entre les formes verbales des deux langues.

En outre, nos résultats mettent particulièrement en évidence que l'influence du penser en L1 pour parler en L2 (voir exemples [1a]-[1c] ; transfert conceptuel, Jarvis et Pavlenko, 2010) est encore une fois plus manifeste chez les sujets anglais qui apprennent cette fois-ci l'italien, mais uniquement aux stades avancés et seulement pour des phénomènes spécifiques, à savoir dans l'emploi de particules directionnelles extraverbales (VS en italien). Ces résultats vont à l'encontre de ceux de Cadierno (2004) et de Cadierno et Ruiz (2006), qui insistent sur l'impact important de la L1 dans le discours en L2 chez les apprenants intermédiaires. Nos apprenants anglophones, en effet, n'essaient pas d'encoder la manière plus que les autres groupes d'apprenants; en revanche, ils exploitent de manière conséquente les constructions satellitaires en italien (emploi de VS tels que correre via, andare via), contrairement aux francophones pour la même LC, alors qu'ils n'essaient pas de réaliser de telles constructions en français L2. Le transfert conceptuel ainsi relevé chez les anglophones avancés est sans doute lié au fait qu'à ce stade ils disposent de plus de moyens que les intermédiaires pour la mise en mots d'une conceptualisation typique de leur L1 
dans la variété cible (voir Stutterheim, 2003, concernant l'attention prêtée au point final ou au déroulement de l'action; Carroll et Stutterheim, 1997, Carroll et al., 2000 et Carroll et Lambert, 2006, pour la construction des chaînes anaphoriques dans le discours). Mais comment justifier le rôle joué par la L1 seulement chez ce groupe d'apprenants et pour cette LC?

Le contraste attesté dans les productions des anglophones en italien L2 vs français L2 montre bien que le transfert n'a lieu que si la LC l'encourage, notamment grâce à la présence de constructions formellement similaires à celles de la L1 (principe du transfer to somewhere, Andersen, 1983). Il est toutefois pertinent de discuter les raisons possibles du transfert «tardif» des structures satellitaires en italien L2.

Nos résultats semblent refléter à la fois l'orientation vers les moyens de la LC, déjà constatée chez les apprenants débutants du projet ESF (voir Perdue, 1993b; Becker et Carroll, 1997), et le principe de la recherche des similarités entre les langues en présence, tel qu'il a été défini par Ringbom et Jarvis (2009). En effet, malgré son statut de langue à dominante satellitaire, l'anglais dispose également des schémas à cadrage verbal (the frog exits the jar, trajectoire dans le verbe). L'italien, de son côté, est une langue dans laquelle le cadrage verbal est dominant, mais qui présente aussi une option à cadrage satellitaire très productive contrairement au français (voir Fagard, 2019), du moins pour l'expression des certaines situations de déplacement et surtout à l'oral. En continuité avec ce qui a été attesté chez les débutants du projet ESF, les apprenants anglophones de l'italien s'orienteraient initialement vers les schémas dominants de la LC, aussi bien en français qu'en italien L2. L'apparition plus tardive des VS en italien L2, malgré l'existence de structures similaires entre les deux langues, pourrait être attribuée à l'action convergente de différents facteurs. Plus précisément, il est possible que les constructions satellitaires de l'italien soient initialement ressenties comme trop «semblables» aux constructions typiques de l'anglais L1: l'évaluation de l'italien en tant que langue typologiquement éloignée ferait obstacle à la transférabilité de structures pourtant similaires entre les deux langues (voir psychotypologie de Kellerman, 1979; Kellerman et Sharwood Smith, 1986). Mais il se peut aussi que l'apparition tardive des VS soit reliée à leur fréquence d'emploi sans doute plus faible dans l'input, par rapport à celle des structures équivalentes à cadrage verbal, surtout pour des sujets qui apprennent la L2 principalement en milieu institutionnel dans leur pays natal. Dans les deux cas, malgré la forte proximité génétique entre le français et l'italien (les deux étant des langues romanes), les productions des anglophones avancés sont plus proches des préférences natives en italien L2 que celles des francophones, du moins en ce qui concerne l'expression du déplacement.

La tendance à chercher des similarités entre les langues semble également expliquer le parcours des apprenants de langue romane. Pour les italophones apprenants du français, il s'agit d'identifier les constructions à cadrage verbal qui sont dominantes aussi bien dans leur LS que dans la LC: l'input (surtout institutionnel pour les sujets de notre corpus) confirme les attentes de similarité pour ce type de constructions et infirme rapidement les attentes possibles de constructions 
apparentées aux VS. De même, les francophones retrouvent rapidement le schéma de constructions à cadrage verbal typiques de leur L1. Une fois cette similitude constatée, ces derniers ne vont pas plus loin dans la recherche d'autres moyens pour exprimer les mêmes concepts (notamment les VS). En effet, il a déjà été remarqué que les apprenants d'une L2 proche tardent à employer des moyens alternatifs spécifiques de la LC, lorsqu'ils diffèrent de leur L1, par rapport à des apprenants avec des L1 plus éloignées (voir Benazzo et Andorno, 2017, concernant l'emploi d'adverbes temporels en français L2 par des italophones vs germanophones).

En somme, les résultats présentés permettent d'approfondir certaines réflexions déjà abordées dans la littérature, notamment autour de la notion de transfert conceptuel lors de l'acquisition d'une L2. En effet, il a été possible de mieux comprendre l'étendue de l'empreinte de la L1 dans la mesure où celle-ci est également liée à d'autres facteurs, tels que le niveau de maîtrise de la L2, ainsi que les caractéristiques typologiques de la LC. En d'autres termes, la présence/absence de structures formellement équivalentes entre la LS et la LC (par ex., dans notre étude, absence de verbes à particules en français, mais présence de ceux-ci en italien pour les apprenants anglophones) peut favoriser ou bloquer un transfert chez l'apprenant avancé, indépendamment du degré variable de proximité/distance typologique en termes spatiaux entre les langues en présence.

\section{Références bibliographiques}

Anastasio, S. 2017. Tra "Thinking for Speaking" e variazioni intratipologiche: lo spazio dinamico in italiano, francese e inglese L1. In A. De Meo et F. M. Dovetro (éd.), La comunicazione parlata/Spoken Communication. Rome: Aracne Editrice: 49-64.

Anastasio, S. 2018. L'expression de la référence à l'espace en italien et en français L2. Une étude comparative. Thèse de doctorat non publiée en sciences du langage. Université Vincennes-Saint-Denis - Paris 8.

Andersen, R. 1983. Transfer to Somewhere. In S. Gass et L. Selinker (éd.), Language Transfer in Language Learning. Rowley: Newbury House: 177-201.

BARTNING, I. 1997. L'apprenant dit avancé et son acquisition d'une langue étrangère. Tour d'horizon et esquisse d'une caractérisation de la variété avancée. AILE-Acquisition et interaction en langue étrangère 9: 9-50. En ligne à l'adresse suivante: https://journals. openedition.org/aile/1316.

Bartning, I. 2009. The Advanced Learner Variety: 10 Years Later. In E. LABEAu et F. MYLes (éd.), The Advanced Learner Variety: The Case of French. Berne: P. Lang: 11-40.

BARTNING, I. et SCHLyTER, S. 2004. Itinéraires acquisitionnels et stades de développement en français L2. Journal of French Languages Studies 14 (3) : 281-299.

Beavers, J., Levin, B. et Wei Tham, S. 2010. The Typology of Motion Expressions Revisited. Journal of Linguistics 46 (2): 331-377.

Becker, A. et Carroll, M. (éd.) 1997. The Acquisition of Spatial Relations in a Second Language. Amsterdam - Philadelphie: J. Benjamins. 
Benazzo, S., Andorno, C., Interlandi, G. et Patin, C. 2012a. Perspective discursive et influence translinguistique. Exprimer le contraste d'entité en français et italien L2. LIA - Language, Interaction and Acquisition 3 (2): 173-201.

Benazzo, S. et Andorno, C. 2017. Is It Really Easier to Acquire a Closely-Related Language? A Study on the Expression of Iteration and Continuation in L2 French. In M. Howard et P. Leclerce (éd.), Tense-Aspect-Modality in a Second Language: Contemporary Perspectives. Amsterdam - Philadelphie: J. Benjamins : 105-143.

Benazzo, S., Flecken, M. et Soroli, E. (éd.) 2012b. Typological Perspectives on Second Language Acquisition: "Thinking for Speaking" in L2. LIA - Language, Interaction and Acquisition 3 (2): 163-172.

Berman, R. A. et Slobin, D. I. (éd.) 1994. Relating Events in Narrative. Hillsdale: L. Erlbaum. Vol. 1: A Crosslinguistic Developmental Study.

Bernini, G., Spreafico, L. et Valentini, A. 2006. Acquiring Motion Verbs in a Second Language: The Case of Italian L2. Linguistica e Filologia 23 : 7-26.

BoriLlo, A. 1998. L'espace et son expression en français. Gap - Paris: Ophrys.

Cadierno, T. 2004. Expressing Motion Events in a Second Language: A Cognitive Typological Perspective. In M. Achard et S. NiEmeIER (éd.), Cognitive Linguistics, Second Language Acquisition, and Foreign Language Teaching. Berlin: De Gruyter: 13-50.

Cadierno, T. 2017. Thinking for Speaking about Motion in a Second Language. In I. IbARRETXe-AntuŇAno (éd.), Motion and Space across Languages: Theory and Applications. Amsterdam - Philadelphie: J. Benjamins: 279-300.

Cadierno, T. et Ruiz, L. 2006. Motion Events in Spanish L2 Acquisition. Annual Review of Cognitive Linguistics 4 : 183-216.

Cardini, F.-E. 2008. Manner of Motion Saliency: An Inquiry into Italian. Cognitive Linguistics 19 (4) : 533-569.

Carroll, M. et Lambert, M. 2006. Reorganizing Principles of Information Structure in Advanced L2s: French and German Learners of English. In H. Byrnes, H. D. WegerGuntharp et K. A. Sprang (éd.), Educating for Advanced Foreign Language Capacities: Constructs, Curriculum, Instruction, Assessment. Washington: Georgetown University Press : 54-73.

Carroll, M., Murcia-Serra, J., Watorek, M. et Bendiscioli, A. 2000. The Relevance of Information Organization to Second Language Acquisition Studies: The Descriptive Discourse of Advanced Adult Learners of German. Studies in Second Language Acquisition 22 (3) : 441-466.

Carroll, M. et Stutterheim, C. von 1997. Relations entre grammaticalisation et conceptualisation et implications sur l'acquisition d'une langue étrangère. AILE Acquisition et interaction en langue étrangère 9: 83-115. En ligne à l'adresse suivante: https://journals.openedition.org/aile/726.

Carroll, M., Weimar, K., Flecken, M., Lambert, M. et Stutterheim, C. von 2012. Tracing Trajectories. Motion Event Construal by Advanced L2 French-English and L2 French-German speakers. LIA - Language, Interaction and Acquisition 3 (2): 202-230.

Cini, M. (éd.) 2008. I verbi sintagmatici in italiano e nelle varietà dialettali. Stato dell'arte e prospettive di ricerca. Francfort-sur-le-Main: P. Lang. 
Dietrich, R., Klein, W. et Noyau, C. 1995. The Acquisition of Temporality in a Second Language. Amsterdam - Philadelphie: J. Benjamins.

Eckman, F. 1996. A Functional-Typological Approach to Second Language Acquisition Theory. In W. C. Ritchie et T. K. Bhatia (éd.), Handbook of Second Language Acquisition. San Diego - New York - Boston: Academic Press : 195-211.

FAgARD, B. 2019. From "il s'envole hors" to "il sort du nid": A Typological Change in French Motion Expressions. In M. Aurnague et D. Stosic (éd.), The Semantics of Dynamic Space in French. Descriptive, Experimental and Formal Studies on Motion Expression. Human Cognitive Processing 66. Amsterdam - Philadelphie: J. Benjamins : 110-138.

Han, Z. et CAdierno, T. 2010. Linguistic Relativity in Second Language Acquisition: Thinking for Speaking. Bristol - Buffalo: Multilingual Matters.

Hendriks, H. et Hickmann, M. 2011. Expressing Voluntary Motion in a Second Language: English Learners of French. In V. J. Cоок et B. BAssetTi (éd.), Language and Bilingual Cognition. New York - Hove: Psychology Press: 315-340.

Hendriks, H., Hickmann, M. et Demagny, A.-C. 2008. How Adult English Learners of French Express Caused Motion: A Comparison with English and French Natives. AILE - Acquisition et interaction en langue étrangère 27: 15-41. En ligne à l'adresse suivante: https://journals.openedition.org/aile/3973.

Hickmann, M. 2012. Diversité des langues et acquisition du langage: espace et temporalité chez l'enfant. Langages $188: 25-39$.

Hickmann, M., Hendriks, H., Demagny, A.-C., Engeman, H., Iakovleva, T., Ji, Y., OchSenbauer, A. K. et Soroli, E. 2014. La représentation de l'espace-études expérimentales, translinguistiques et développementales. Vol. 2: Annexes. Manuel non publié.

IACOBInI, C. et FAGARD, B. 2011. A Diachronic Approach to Variation and Change in the Typology of Motion Event Expression. A Case Study: From Latin to Romance. Faits de langues - Les cabiers 3: 151-172.

Iacobini, C. et Masini, F. 2007. Verb-Particle Constructions and Prefixed Verbs in Italian: Typology, Diachrony and Semantics. In G. Booij, L. Ducceschi, B. Fradin, E. Guevara, A. Ralli et S. Scalise (éd.), On-line Proceedings of the Fifth Mediterranean Morphology Meeting - MMM5 (Fréjus, 15-18 September 2005). Bologne: Università degli Studi di Bologna: 157-184. En ligne à l'adresse suivante: https://geertbooij.files.wordpress. com/2014/02/mmm5-proceedings.pdf.

Ibarretxe-Antuñano, I. 2009. Path Salience in Motion Events. In J. Guo, E. Lieven, N. Budwig, S. Ervin-Tripp, K. Nakamura et S. ÔzÇAlişKan (éd.), Crosslinguistic Approaches to the Psychology of Language: Research in the Tradition of Dan Isaac Slobin. New York: Psychology Press: 403-414.

IbarretXe-Antuñano, I. 2015. Going beyond Motion Events Typology: The Case of Basque as a Verb-Framed Language. Folia Linguistica 49 (2) : 307-352.

IbarRetXe-Antuñano, I. et Hijazo-Gascón, A. 2012. Variation in Motion Events. Theory and Applications. In L. Filipović et K. M. Jaszczolt (éd.), Space and Time in Languages and Cultures. Linguistic Diversity. Amsterdam - Philadelphie: J. Benjamins: 349-371. 
Ibarretxe-Antuñano, I. et Hijazo-Gascón, A. 2013. Same Family, Different Paths: Intratypological Differences in Three Romance Languages. In J. Goschler et A. Stefanowitsch (éd.), Variation and Change in the Encoding of Motion Events. Amsterdam - Philadelphie: J. Benjamins : 39-54.

IbarretXe-Antuñano, I., Hijazo-Gascón, A. et Moret-Olivier, M.-T. 2017. The Importance of Minority Languages in Motion Event Typology. The Case of Aragonese and Catalan. Motion and Space across Languages. Amsterdam - Philadelphie: J. Benjamins: 123-149.

Jarvis, S. et Pavlenko, A. 2010. Crosslinguistic Influence in Language and Cognition. Londres - New York: Routledge.

Kellerman, E. 1979. La difficulté, une notion difficile. Encrages (numéro spécial de linguistique appliquée) : 16-21.

Kellerman, E. et Sharwood Smith, M. (éd.) 1986. Crosslinguistic Influence in Second Language Acquisition. New York - Oxford - Toronto: Pergamon Institute of English.

Klein, W. et Perdue, C. 1997. The Basic Variety (or: Couldn't Natural Languages Be Much Simpler?). Second Language Research 13 (4): 301-347.

Kopecka, A. 2006. The Semantic Structure of Motion Verbs in French: Typological Perspectives. In M. Hickmann et S. Robert (éd.), Space in Languages. Linguistic Systems and Cognitive Categories. Amsterdam - Philadelphie: J. Benjamins: 83-101.

Kopecka, A. sous presse. From a Satellite- to a Verb-Framed Pattern: A Typological Shift in French. In H. Cuyckens, W. De Mulder et T. Mortelmans (éd.), Variation and Change in Adpositions of Movement. Amsterdam - Philadelphie: J. Benjamins.

Levinson, S. C. 2003. Space in Language and Cognition: Explorations in Cognitive Diversity. Cambridge - New York: Cambridge University Press.

MacWhinney, B. 2000. The CHILDES Project: Tools for Analyzing Talk. Mahwah Londres: L. Erlbaum [3 $3^{\mathrm{e}}$ éd.].

MaYer, M. 1969. Frog, Where Are You? New York: Dial Books for Young Readers.

Moncó TARACENA, S. 2013. Les verbes syntagmatiques italiens: éléments contrastifs et didactiques. Epilogos 3: 65-85. En ligne à l'adresse suivante: http://eriac.univ-rouen. fr/wp-content/uploads/2014/04/09PEDLAp65MoncoTaracena.pdf.

Perdue, C. (éd.) 1993a. Adult Language Acquisition: Cross-Linguistic Perspectives. Cambridge : Cambridge University Press.

Perdue, C. 1993b. Comment rendre compte de la "logique" de l'acquisition d'une langue étrangère par l'adulte. Études de linguistique appliquée 92: 8-22.

Perdue, C. 2002. Development of L2 Functional Use. In V. J. Cook (éd.), Portraits of the L2 User. Clevedon - Buffalo - Toronto: Multilingual Matters : 121-144.

Ragnasdottir, H. et Strömevist, S. 2004. Time, Space and Manner in Icelandic and Swedish. In S. Strömqvist et L. Verhoeven (éd.), Relating Events in Narrative. Mahwah: L. Erlbaum. Vol. 2 : Typological and Contextual Perspectives: 113-141.

Ringbom, H. et Jarvis, S. 2009. The Importance of Cross-Linguistic Similarity in Foreign Language Learning. In M. H. Long et C. J. Doughty (éd.), The Handbook of Language Teaching. Oxford - Malden - Chichester: Wiley-Blackwell: 106-118. 
SChwARZe, C. 1985. "Uscire" e "andare fuori" : struttura sintattica e semantica lessicale. In A. Franchi De Bellis et L. M. Savoia (éd.), Sintassi e morfologia della lingua italiana d'uso. Teorie e applicazioni descrittive. Rome: Bulzoni: 355-371.

SHARPEN, R. 2016. L1 Conceptual Transfer in the Acquisition of L2 Motion Events in Spanish and English: The Thinking-for-Speaking Hypothesis. Open Linguistics 2: 235-252.

Simone, R. 1997. Esistono verbi sintagmatici in italiano? In T. De Mauro et V. Lo Cascio (éd.), Lessico e grammatica. Teorie linguistiche e applicazioni lessicografiche. Rome: Bulzoni : 47-61.

Simone, R. 2008. Verbi sintagmatici come costruzione e come categoria. In M. Cini (éd.), I verbi sintagmatici in italiano e nelle varietà dialettali. Stato dell'arte e prospettive di ricerca. Francfort-sur-le-Main: P. Lang: 13-30.

SLOBIN, D. 1996. From “Thought and Language" to "Thinking for Speaking”. In J. GuMPERZ et S. Levinson (éd.), Retbinking Linguistic Relativity. Cambridge: Cambridge University Press : 70-96.

Slobin, D. 2000. Verbalized Events: A Dynamic Approach to Linguistic Relativity and Determinism. In S. Niemeier et R. Dirven (éd.), Evidence for Linguistic Relativity. Amsterdam - Philadelphie: J. Benjamins: 107-138.

Slobin, D. 2004. The Many Ways to Search for a Frog: Linguistic Typology and the Expression in Motion Events. In S. StRÖMQvist et L. Verhoeven (éd.), Relating Events in Narrative. Mahwah: L. Erlbaum. Vol. 2: Typological and Contextual Perspectives: 219-257.

SLobin, D. 2006. What Makes Manner of Motion Salient? Explorations in Linguistic Typology, Discourse and Cognition. In M. Hickmann et S. Robert (éd.), Space in Languages. Linguistic Systems and Cognitive Categories. Amsterdam - Philadelphie: J. Benjamins: 59-81.

Soroli, E. et Verkerk, A. 2017. Motion Events in Greek. Methodological and Typological Issues. CogniTextes - Revue de l'Association française de linguistique cognitive 15: 1-54. En ligne à l'adresse suivante: http://journals.openedition.org/cognitextes/889.

Spreafico, L. 2008. Tipologie di lessicalizzazioni adverbiali in alcune lingue d'Europa. In M. Cini (éd.), I verbi sintagmatici in italiano e nelle varietà dialettali. Stato dell'arte e prospettive di ricerca. Francfort-sur-le-Main: P. Lang: 61-81.

Spreafico, L. 2009. Problemi di tipologia lessicale. I verbi di moto nello Standard Average European. Rome: Bulzoni.

Spreafico, L. et VAlentini, A. 2009. Gli eventi di moto: strategie di lessicalizzazione nell'italiano di nativi e di non nativi (immigrati o in mobilità). Segundas Lenguas e Inmigración en red 3: 66-87. En ligne à l'adresse suivante: https://dialnet.unirioja.es/ descarga/articulo/3186898.pdf.

Stutterheim, C. von 2003. Linguistic Structure and Information Organisation. The Case of Very Advanced Learners. In S. H. Foster-Cohen et S. Pekarek Doehler (éd.), EUROSLA Yearbook. Amsterdam - Philadelphie: J. Benjamins. Vol. 3: 183-206.

TAlmy, L. 1985. Lexicalization Patterns: Semantic Structure in Lexical Forms. In T. SHOPEN (éd.), Language Typology and Syntactic Description. Cambridge: Cambridge University Press. Vol. 3: Grammatical Categories and the Lexicon: 57-149. 
Talmy, L. 2000. Toward a Cognitive Semantics. Cambridge: MIT Press.

Verkerk, A. 2015. Where Do All the Motion Verbs Come From? The Speed of Development of Manner Verbs and Path Verbs in Indo-European. Diacbronica 32 (1): 69-104.

Watorek, M. 1996. Le traitement prototypique: définitions et implications. Toegepaste Taalwetenschap in Artikelen 55 : 187-200. 